\title{
Event-related brain potentials during orienting to auditory and visual stimulation in spinal cord injured humans
}

\author{
M J Cohen PhD, ${ }^{1,2} \mathrm{~S}$ L Schandler PhD, ${ }^{1,3}$ M Vulpe $\mathrm{MD}^{4,5}$ \\ ${ }^{1}$ Research Service, Department of Veterans Affairs Medical Center, 5901 E Seventh Street, \\ Long Beach, CA 90822, USA; ${ }^{2}$ Department of Psychiatry and Human Behavior, \\ University of California, Irvine; ${ }^{3}$ Division of Psychology, Chapman University; ${ }^{4}$ Spinal \\ Cord Injury Service, Department of Veterans Affairs Medical Center, Long Beach; \\ ${ }^{5}$ Department of Neurology, University of California, Irvine, California, USA.
}

In intact humans, deprivation of somatosensory and kinesthetic sensations result in significant alterations in perception and information processing. There have been very few studies to discover if the loss of sensation with spinal cord injury (SCI) in humans affects perceptual operations. We hypothesized that the SCI participant would either exhibit arousal, perceptual, and information processing alterations similar to experimentally sensory deprived subjects (who provide the closest human analogue), or that the somatosensory cortex would show reorganization for the processing of other modalities of stimulation.

The subjects consisted of 16 paraplegic, 13 quadriplegic, and 22 non SCI controls. Subjects received an auditory orienting task consisting of a $500 \mathrm{~Hz}$ tone presented 20 times each at $66,75,88$, and $101 \mathrm{db}$ and a visual orienting task incorporating light flashes of 115, 123, 131, and 140 lux presented 20 times each. EEG information processing data were recorded from $\mathrm{C} 3$ and $\mathrm{C} 4$ for $100 \mathrm{msec}$ prior to and $500 \mathrm{msec}$ post stimulation

Information processing variables, analyzed as event-related potentials, indicated that the somatosensory cortex of SCI groups had a flattened response to auditory stimulation. The control group had a significantly larger P2 component. We concluded that these data signified that the somatosensory cortex did not reorganize function in response to chronic deafferentation nor was the SCI subject hyperresponsive to stimulation.

Keywords: spinal cord injuries; event-related potentials; cortical reorganization; orienting.

\section{Introduction}

Depending on its level and severity, spinal cord injury (SCI) produces moderate to profound loss of somatosensory, kinesthetic and proprioceptive experience. The loss of movement and sensation and the deafferentation of the somatosensory cortex should have a major impact on the injured person's perceptual and cognitive processes. The theories and data from sensory deprivation experiments provide a model for predicting the nature of these perceptual changes for acute sensory and kinesthetic loss, but may have little predictive power for chronic somatosensory deafferentation. Evidence is increasing that neuronal connections can be modified because of experience and environmental stimuli and these modifications can occur in either a transient or permanent manner. These findings may help to explain functional changes in the somatosensory cortex of chronic SCI patients.

Experimental sensory deprivation (SD) studies have produced fairly consistent changes in dominant brain rhythms. Zuckerman and Hopkins ${ }^{1}$ found that after the first hour of SD the electroencephalogram (EEG) slowed from an excited beta pattern to a presleep pattern. Heron ${ }^{2,3}$ studied 6 subjects who each spent 4 days in an isolation chamber. As the deprivation period grew long, slowed alpha waves of 
high voltages and delta wave activity increased. Several additional studies have confirmed these findings. ${ }^{4-6}$

Perceptual and performance alterations due to deprivation of a single sensory system, especially tactual or kinesthetic deprivation, are of particular interest for our understanding of the effects of SCI-induced sensory deprivation on perception. Increased tactual sensitivity has been found following prolonged tactual deprivation. Early studies from the McGill University laboratories ${ }^{3,7}$ occluded the volar surface of the forearm for 4 days; subsequent testing with von Frey hairs revealed greater sensitivity for the experimental area following the occlusion.

Extending this work, Aftanas and Zubek $^{8.9}$ studied changes in heat pain threshold and tactual acuity following 7 days of no tactile stimulation, constant pressure, or normal stimulation. Though no changes in heat pain threshold were found, they reported significantly increased tactual sensitivity for the no stimulation group, significantly decreased tactual sensitivity for the constant pressure group, and no change for the control group. These effects, persisting for up to 6 days, argue in favor of CNS alterations in perception rather then local mechanisms such as impaired circulation. Normal circulation would have been restored in less time. Zubek ${ }^{10}$ argued that these results demonstrated that tactile deprivation caused changes in primary somatosensory cortex analogous to Stavraky's ${ }^{11}$ findings in monkey of supersensitivity in the sensory cortex following partial deafferentation at lower levels of the CNS. Zubek proposed that experimental tactual deprivation may produce a temporary functional state of deafferentation. These data showing long-lasting supersensitivity suggest a reorganization of the deafferentated somatosensory cortex.

Though we found no studies testing perceptual changes following tactual deprivation, several experiments have been published on perceptual change following kinesthetic deprivation. Using a battery of 7 perceptual-motor tasks, Zubek et al ${ }^{12}$ found that one day of immobilization produced significant impairment of dexterity, kines- thetic acuity, color discrimination, and performance on a reversible figures test. Unaffected were pain sensitivity, tactual acuity, and cancellation of numbers. These findings were extended to a one-week immobilization period. Zubek ${ }^{10}$, reporting data from a subexperiment testing for visual and auditory vigilance, was surprised to find that the immobilized condition produced increased visual vigilance.

Because spinal cord injured people have moderate to profound loss of tactual and/or kinesthetic sensation, we expected they would act similarly to subjects experiencing experimental SD, especially deprivation of tactual and/or kinesthetic stimulation. One area of comparison would be changes in physiological indications of activation. The few studies of physiologic arousal with SCI subjects are limited to EEG dependent variables. Several investigators ${ }^{13-15}$ point to disruptions in normal EEG patterns, but results are inconsistent.

Only three experiments assessing information processing in SCI patients were found. Hester ${ }^{16}$ compared 20 functionally complete SCI patients (14 cervical and 6 thoracic lesions) with 20 controls on a verbal serial coding task and a reaction time task each under low, medium, and high incentives. Incentive level was proposed as a means of varying cortical arousal. No differences were found between the groups on any task-incentive combination. Positive results are provided in the relatively recent studies by Richards. ${ }^{15,17}$ Fifteen quadriplegic inpatients, 7 paraplegic inpatients, 4 quadriplegic outpatients and 16 non SCI controls performed an auditory vigilance task. ${ }^{15}$ There were no task related differences between SCI and control subjects, and EEG arousal states measured during the task were similar. When subsets of the SCI group were compared, inpatients performed worse than outpatients, and recently injured patients had poorer performance and lower EEG-measured arousal than subjects with older injuries. Richards et $a l^{17}$ assessed auditory perception in 6 quadriplegic outpatients and 12 controls using a dichotic listening task while recording auditory evoked potentials (EP) and auditory thresholds. No group differences were 
found on measures of auditory threshold or auditory EP, which the authors suggested indicated that the SCI group had an intact auditory pathway. The SCI group exhibited a significant superiority in aspects of the dichotic listening task. For example with double-word presentations, the SCI group was consistently more accurate than the unimpaired control group. The investigators argued that the data reflects attention-motivational differences between able bodied and SCI participants because the SCI individual is, in a sense, stimulus starved, thus more attentive to external stimuli. These data agree with Zubek's ${ }^{10}$ report that several days of experimentally produced immobilization increased vigilance behavior.

An alternative explanation is that the cortex, now deprived of somatosensory input, reorganises to process other modalities of sensory information. For example, Merzenich et al $^{18}$ found that in monkey, following amputation of one or two fingers, the somatosensory cortical map progressively changed over several months. The cortical representations of adjacent digits and the palmar surface slowly expanded until they occupied most of the area previously represented by the amputated digits. While adjacent areas may invade fairly small zones of deafferentated somatosensory cortex, it is unlikely to fully invade the massive deafferentated areas caused by a spinal cord injury. In such cases, the somatosensory cortex might reorganize to perform a new sensory function. Neville and colleagues ${ }^{19,20}$ have reported that, in congenitally deaf individuals, the auditory cortex reorganised to process visual stimulation. The authors described data from a group of deaf individuals with larger event-related potentials over both visual and auditory cortex to visual stimuli compared to control subjects.

A sensory deprivation model of spinal cord injury predicts that SCI individuals will have reduced cortical arousal, increased peripheral arousal, and changes in perception and performance similar to individuals experimentally sensory deprived. A very limited body of research with SCI patients has produced conflicting results leaving the veracity of the sensory deprivation model in doubt. A hypothesis of reorganization of the somatosensory cortex predicts that, compared to controls, event-related potentials from SCI groups would be larger when processing auditory or visual information. We tested the predictions of these models by measuring somatosensory cortical arousal (EEG event-related potentials) of paraplegics, quadriplegics and controls, while participants passively listened to tones of various intensities. The paradigm selected has well known effects on the P2 component of the ERP. ${ }^{21,22}$ With somatosensory stimulation, the $\mathrm{P} 2$ component is generated in the primary somatosensory cortex. ${ }^{23}$ If the cortex has reorganized to process other modalities, such as auditory or visual information, the $\mathrm{P} 2$ component should be larger in the SCI participants.

\section{Methods}

\section{Subjects}

Sixteen paraplegic, 13 quadriplegic, and 22 control subjects were each paid $\$ 20.00$ for voluntary participation in the experiment. Inclusion criteria were (a) age between 21 and 65 years old and a negative history for (b) major psychiatric illness, (c) alcohol abuse or drug addiction, (d) chronic use of a major psychotropic drug. The SCI participants were recruited from the clinics of the Spinal Cord Injury Service at the Department of Veterans Affairs Medical Center, Long Beach. Additional inclusion criteria for the SCI volunteers were negative histories for (a) a major medical problem that was not secondary to the SCI or considered severe for the SCI population, (b) peripheral neuropathy, (c) cardiac arrhythmias, (d) cardiac or hepatic dysfunction. (d) renal insufficiency, (e) head injury or seizures, or (f) recurrent autonomic dysreflexia. The control volunteers, recruited from the staff of the Medical Center and from the population of California State University, Long Beach, were in generally good health and matched for age and sex to the SCI groups.

\section{Apparatus}

The dependent variables were measured with a Grass model 7D polygraph. The polygraph was linked to an AST premium 
286 computer which contained a Data Translation DT2821 analog and digital I/O system. Analog-to-digital conversion and control of the experimental environment were done with the Asyst version 3.1 programming language. A two-channel EEG was obtained from an Electrode Caps International Electro-Cap System with $\mathrm{Pb}$ electrodes positioned at $\mathrm{C}_{3}$ and $\mathrm{C}_{4}$. Each electrode site was abraded with a blunt, sterile needle and filled with Electrode Caps International electrode gel. Linked ears served as the reference. Eye blinks and eye movements were detected from a $3 \mathrm{~mm}$ Beckman $\mathrm{Ag} / \mathrm{AgCl}$ electrode placed slightly inferior to the right external canthus and referenced to a similar electrode at the nasion. After 15 min of stabilization, electrode impedances were evaluated with a Grass EZM5A impedance meter. Electrodes with impedances above $10 \mathrm{~K} \Omega$ were reapplied. The electrodes for EEGs and eye movements were directed through Grass 7HIP5 high impedance probes to 7P3C AC preamplifiers with bandpass filtering set at 0.1 and $75 \mathrm{~Hz}$. An electrocardiogram and skin conductance were also obtained, but the data are not reported in this manuscript.

Auditory stimulation, consisting of 500 $\mathrm{Hz}, 1.0 \mathrm{sec}$ duration tones of $62,75,88$, and $101 \mathrm{~dB}$ SPL, was generated by a Coulbourn S81-06 precision signal generator, S85-08 attenuator, S84-04 rise fall gate, and S82-24 audio mixer amplifier connected to Realistic Nova 40 earphones. Rise and fall times were $200 \mathrm{msec}$. Visual stimuli were generated by a Grass P-22 visual stimulator placed 1 meter from the subject's head. Light flashes of $10 \mu \mathrm{sec}$ duration were calibrated to 115 , 123,131 , and 140 lux with a Grossen Emulex 2 meter.

\section{Procedure}

Following medical screening, those persons acceptable for participation were seen individually at the laboratory. After signing an informed consent form approved by the Medical Center's Human Studies Subcommittee, electrodes were attached for recording dependent variables. A 15 min electrode stabilization period ensued during which a brief audiometry test, using ascending and descending series of $500 \mathrm{~Hz}$ tones, determined that each participant had near normal hearing level. Following a 10 minute restadaptation period, a subject received 20 presentations each of 4 tone intensities $(62$, $75,88,101 \mathrm{~dB}$ SPL) or flash intensities (115, $123,131,140$ lux ) on inter-trial intervals of $13,16,19$, or $22 \mathrm{sec}$. The stimuli were presented in 5 different greco-latin squares. Within a square, each stimulus intensity was paired with each inter-trial interval one time. Whether a participant received auditory or visual stimuli first was determined randomly and a 2 min rest period intervened between stimulus modalities.

\section{Measurement and averaging of dependent variables}

EEGs were digitized at 200 samples/sec for $100 \mathrm{msec}$ pre and $1000 \mathrm{msec}$ post stimulation. This resulted in 220 digital values per channel for each stimulus trial. Eye movement-contaminated EEG trials were removed by a computer algorithm. A technician checked the accuracy of the algorithm on a random $10 \%$ of the trials. The computer also rejected any trial with absolute amplitudes exceeding $100 \mu \mathrm{V}$. On average, less than $10 \%$ of the total trials were rejected. ERP responses were averaged over the 20 trials at each intensity for the $500 \mathrm{msec}$ post stimulation epoch. The sampling period was baseline zeroed by subtracting out the average of the $100 \mathrm{msec}$ prestimulus epoch. To detect peaks and latencies of major components of the ERP, a computer program recorded maximum positivity for the P1 $(30-80 \mathrm{~ms})$ and P2 (130-380 ms), and maximum negativity for the N1 (70-150 ms) and N2 (180-350 ms) along with the times these maxima occurred.

\section{Results}

The probability level chosen for protection against Type I errors was $p<0.05$. All analyses were performed on a PC 486 computer using the BMDP/386 statistical software package. Analysis of variance (ANOVA) revealed no differences among 
the groups for age and no difference between the paraplegic and quadriplegic groups for length of time since injury. ANOVA reported no differences between comparable components at the $\mathrm{C} 3$ and $\mathrm{C} 4$ sites for either auditory or visual stimuli; thus the data were summed over recording sites for subsequent analyses. Figure 1 shows the grand averaged ERPs for each group to the tone stimuli. The data were averaged over trials within a stimulus intensity and then averaged over intensities. The control group conforms to the typical descriptions of sensory ERPs to auditory stimuli where the subject passively attends to stimulation. ${ }^{21,22}$ The group had well formed components and, as expected, the $\mathrm{N} 1-\mathrm{P} 2$ increased in amplitude as the tones become louder. ${ }^{21}$ The patterns of responding for the SCI groups, especially the quadriplegic group, were distinctly different. In general, compared to the control group, the $\mathrm{N} 1-\mathrm{P} 2$ complexes for the $\mathrm{SCI}$ groups were of smaller size especially for the lower amplitude tones. The quadriplegic group exhibited flat, poorly formed response components. Figure 1 also indicates that latencies were longer for N1-P2 components for the quadriplegic group.

Table I lists the mean amplitudes and standard errors of the means for the major

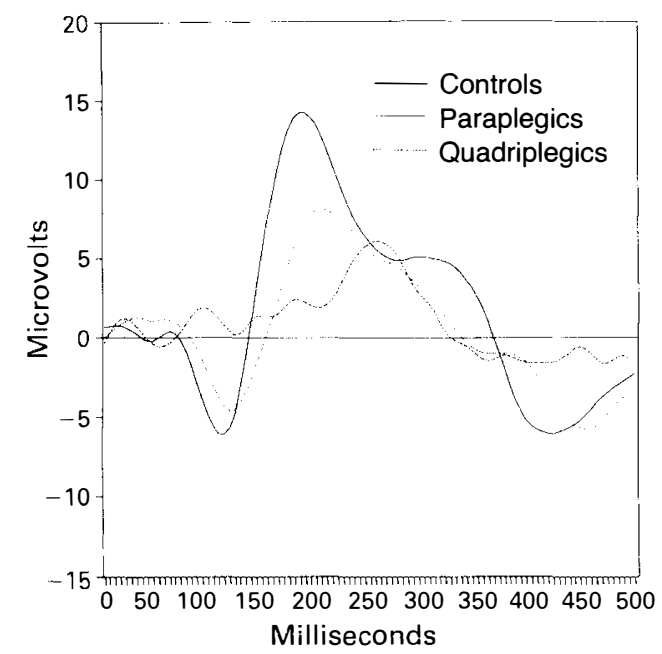

Figure 1 Grand average event-related potentials from control, paraplegic, and quadriplegic groups during auditory stimulation.
ERP components for tones and flashes separately. For each ERP component, 3 (groups) $\times 4$ (stimulus intensities) ANOVAs were performed separately for amplitudes and latencies to auditory and visual stimuli. The analyses for auditory data revealed that the $\mathrm{N} 1, F(3 / 144)=15.96, \mathrm{P} 2$, $F(3 / 144)=13.82$, and N2, $F(3 / 144)=7.06$, amplitude components were sensitive to changes in loudness. The P2 component increased in a linear fashion with increased stimulus loudness $(62 \mathrm{~dB}=11.3 \mu \mathrm{V}, 75 \mathrm{~dB}$ $=15.1 \mu \mathrm{V}, \quad 88 \quad \mathrm{~dB}=17.0, \quad$ and $101 \mathrm{~dB}=24.0 \mathrm{~V})$. A significant group effect was also found for the P2 component, $F(2 / 48)=3.46$ (controls $=22.3 \mu \mathrm{V}$, paraplegic $=14.74 \mu \mathrm{V}, \quad$ and $\quad$ quadriplegic $=$ $13.45 \mu \mathrm{V})$. Tukey HSD a posteriori tests showed a significantly larger $\mathrm{P} 2$ response for the control group compared to the paraplegic or quadriplegic groups. The SCI groups did not differ from each other. Analyses of latencies for the ERP components revealed a significant Groups $\mathrm{X}$ intensity interaction, $F(6,144)=2.39$, for N1. Analysis of simple main events did not indicate a systematic source for the interaction effect. Implied differences in latencies for other ERP components seen in Figure 1 were not confirmed statistically.

The visual data had significant effects for all ERP amplitude components due to stimulus intensities, but no group or interactive effects. No significant effects were found for latencies of components to the visual stimuli.

\section{Discussion}

This study assessed if spinal cord injury resulted in either reorganization of the somatosensory cortex for processing of non somatosensory information, or enhanced attention to stimulation because of sensory deprivation. The results indicated differences in the ERPs of spinal cord injured groups compared to a control group. Data from the control group are consistent with other published studies in which the subject passively listened to tones of different loudness or light flashes of differing brightness. The P2 component for the tone data dis- 
Table 1 Event-related potential components to auditory and visual stimulation

\begin{tabular}{|c|c|c|c|c|c|c|}
\hline & \multicolumn{2}{|c|}{ Controls } & \multicolumn{2}{|c|}{ Paraplegics } & \multicolumn{2}{|c|}{ Quadriplegics } \\
\hline & Tones & Flashes & Tones & Flashes & Tones & Flashes \\
\hline & $(\mu \mathrm{V})$ & $(\mu \mathrm{V})$ & $(\mu \mathrm{V})$ & $(\mu \mathrm{V})$ & $(\mu \mathrm{V})$ & $(\mu \mathrm{V})$ \\
\hline \multicolumn{7}{|l|}{$62 \mathrm{~dB}$} \\
\hline P1 & $3.7 \pm 1.5$ & $3.2 \pm 1.3$ & $3.6 \pm 0.9$ & $4.7 \pm 1.3$ & $2.4 \pm 1.8$ & $5.2 \pm 1.4$ \\
\hline N1 & $6.9 \pm 1.7$ & $7.8 \pm 1.5$ & $4.4 \pm 2.2$ & $7.0 \pm 1.8$ & $5.6 \pm 1.4$ & $2.7 \pm 1.7$ \\
\hline P2 & $14.3 \pm 2.3$ & $11.8 \pm 2.3$ & $9.4 \pm 1.5$ & $7.6 \pm 2.4$ & $10.1 \pm 2.5$ & $9.5 \pm 1.5$ \\
\hline $\mathrm{N} 2$ & $11.6 \pm 2.4$ & $8.8 \pm 2.4$ & $7.4 \pm 1.5$ & $8.1 \pm 1.1$ & $9.1 \pm 3.4$ & $2.0 \pm 1.8$ \\
\hline \multicolumn{7}{|c|}{$\begin{array}{c}75 \mathrm{~dB} \\
123 \mathrm{Lux}\end{array}$} \\
\hline P1 & $3.1 \pm 1.7$ & $1.2 \pm 2.0$ & $4.1 \pm 1.6$ & $2.7 \pm 1.5$ & $5.2 \pm 1.6$ & $0.7 \pm 1.5$ \\
\hline N1 & $8.2 \pm 2.0$ & $15.8 \pm 1.8$ & $4.8 \pm 2.3$ & $11.4 \pm 1.8$ & $3.5 \pm 1.1$ & $8.7 \pm 1.9$ \\
\hline P2 & $19.7 \pm 2.5$ & $13.4 \pm 3.1$ & $13.1 \pm 3.3$ & $6.7 \pm 2.6$ & $12.4 \pm 3.1$ & $6.9 \pm 2.3$ \\
\hline $\mathrm{N} 2$ & $10.7 \pm 3.7$ & $12.4 \pm 3.0$ & $8.4 \pm 2.9$ & $8.5 \pm 1.7$ & $8.8 \pm 3.4$ & $7.9 \pm 2.8$ \\
\hline \multicolumn{7}{|c|}{$88 \mathrm{~dB}$} \\
\hline $131 \mathrm{Lu}$ & & & & & & \\
\hline P1 & $4.4 \pm 1.1$ & $1.9 \pm 1.5$ & $5.0 \pm 1.5$ & $0.6 \pm 2.0$ & $5.0 \pm 2.0$ & $1.3 \pm 1.4$ \\
\hline N1 & $8.0 \pm 2.2$ & $15.3 \pm 2.8$ & $7.7 \pm 1.2$ & $8.4 \pm 2.2$ & $5.2 \pm 3.2$ & $7.7 \pm 1.3$ \\
\hline P2 & $22.4 \pm 3.0$ & $12.1 \pm 2.5$ & $14.1 \pm 3.4$ & $7.4 \pm 2.5$ & $14.6 \pm 3.2$ & $6.4 \pm 1.8$ \\
\hline $\mathrm{N} 2$ & $9.9 \pm 3.2$ & $12.4 \pm 2.5$ & $10.9 \pm 2.5$ & $6.2 \pm 2.1$ & $9.1 \pm 3.5$ & $7.1 \pm 2.2$ \\
\hline \multicolumn{7}{|l|}{$101 \mathrm{~dB}$} \\
\hline $140 \mathrm{~L}$ & & & & & & \\
\hline P1 & $5.7 \pm 1.6$ & $0.9 \pm 1.7$ & $5.7 \pm 1.9$ & $1.3 \pm 2.1$ & $5.9 \pm 1.8$ & $1.0 \pm 1.8$ \\
\hline N1 & $16.7 \pm 3.3$ & $14.0 \pm 2.1$ & $15.9 \pm 2.4$ & $10.4 \pm 2.0$ & $12.5 \pm 5.3$ & $7.7 \pm 1.6$ \\
\hline P2 & $33.0 \pm 4.9$ & $17.8 \pm 3.1$ & $22.4 \pm 4.5$ & $12.3 \pm 3.3$ & $16.7 \pm 3.7$ & $8.5 \pm 1.9$ \\
\hline $\mathrm{N} 2$ & $18.7 \pm 4.7$ & $12.7 \pm 3.5$ & $13.2 \pm 3.6$ & $9.4 \pm 2.5$ & $14.3 \pm 3.9$ & $10.4 \pm 2.3$ \\
\hline
\end{tabular}

played sensitivity to the intensity of stimulation. The tone data showed increased amplitudes and shorter latencies to louder tones. The light flashes did not produce significant group differences in the P2 component, but the data did show the control group to have increased P2 amplitude to brighter flashes.

Except for the loudest tone $(101 \mathrm{~dB})$ and the brightest flashes (140 lux), the SCI groups had smaller amplitude ERP components, especially $\mathrm{P} 2$, with nonsignificantly longer response latencies. From a sensory deprivation or stimulus seeking model, we had expected the SCI groups to be hyperresponsive to stimulation. While it is conceivable that hyperresponsiveness could result in undifferentiated ERPs across the stimulus intensities, overall the SCI groups should have had larger ERP components and possibly shorter latencies than the control group. A sensory deprivation model would also predict that the quadriplegic group, because of more profound sensory deprivation, would have larger responses than the paraplegic group. The data indicated no significant differences between the SCI groups which does not support a sensory deprivation model of responding. These findings also do not support a hypothesis of cortical reorganization. If sensory deprived somatosensory cortex in SCI reorganized to process auditory and/or visual information, we would predict larger ERP responses to auditory and/or visual stimulation. We might also predict shorter latencies of those ERP components specifically related to cognitive processing as opposed to stimulus transmission. No analysis found the paraplegic or quadriplegic group to have a heightened component or a shorter latency to either the tones or the flashes.

There are several explanations why the 
SCI groups could have smaller ERP components to auditory and visual stimulation. A simple explanation is that the overall activity level recorded from central electrode positions $(\mathrm{C} 3, \mathrm{C} 4)$ is heavily influenced by activity on the somatosensory cortex. Depending on the level and completeness of an SCI, a large proportion of the somatosensory cortex is deafferentated and, therefore, functionally inactive (though this would support neither a reorganization or deprivation model). If the somatosensory cortex is the source for the differences we see among the groups, recordings from several other electrode sites should not differentiate the groups. We are exploring this possibility in current experiments.

The data are not consistent with the findings of Zubek ${ }^{10}$ and Stavraky. ${ }^{11}$ Zubek hypothesized that the increased sensitivity to tactile stimulation lasting up to 6 days following a week of tactile stimulus deprivation, was caused by a functional supersensitivity in the primary sensory cortex. Zubek drew an analogy to Stavraky's report of supersensitivity in cat somatosensory cortex following partial deafferentation at lower levels of the CNS. While Zubek's and Stavraky's experiments are long term for laboratory research, the length of the sensory deprivation is brief compared to chronic SCI patients. Subjects in our study were seen 18 to 433 months post injury.

Many clinical studies of neural functioning following SCI find, after recovery from spinal shock, hypersensitivity in many systems which stabilizes over time. Additionally, there is evidence that time-since-SCI is correlated with anatomical evidence for retrograde degeneration of neurons up through the somatosensory cortex. ${ }^{24}$ Thus, experimental models based on a week or a month of deprivation may be inappropriate for predicting how chronic SCI sensory deafferentation of months to many years will influence physiological manifestations of information processing.

From a behavioral viewpoint, the data from the SCI groups are consistent with an attention deficit hypothesis. Response patterns to the louder tones $(88$ and $101 \mathrm{~dB}$ ) were more similar to those of the control group than responses to the lower tones. Similar data were found for the light flashes. With respect to the tone data, each person received a brief audiometry test, so we know all individuals had essentially normal hearing levels for $500 \mathrm{~Hz}$ tones. Perhaps the loss of sensory input, including kinesthetic and proprioceptive sensations, causes significantly reduced input to the reticular activating system, resulting in a lowered level of cortical arousal. A result could be inattention to levels of stimulation that are easily processed by an individual with an intact sensory system and normal input to the reticular activating system. If the inattention is functional, it could be tested by pre-cuing the SCI subject about the impending stimulus.

Our findings are more supportive of Hester's ${ }^{16}$ study in which he attempted to behavorially manipulate cortical arousal. $\mathrm{He}$ found no differences between SCI and control participants on either a verbal serial coding task or a reaction time task. Though Richards ${ }^{15.17}$ found some processing superiority for SCI subjects in aspects of a dichotic listening task, no differences were found in either dominant EEG rhythm or auditory EP. The current experiment found smaller ERP components for the SCI groups. Both Hester's and Richard's tasks were more process intensive than our simple, passive listening task. We have suggested that the SCI groups behave electrophysiologically as if they have an attention deficit because ERP components are flat to lower tones and dimmer flashes but more normal to louder tones and brighter flashes. These data would be in agreement with Hester's and Richard's findings in which more complex tasks produced normal responding.

Though the explanations for these interesting findings are speculative, they are testable. Running SCI participants through paradigms that manipulate the behavioral significance of the stimuli should clarify the relationship between the alterations in ERP components and the person's ability to properly process the information contained in the stimulus. Our audiometry test data indicate that SCI individuals hear as well as our controls, but the SCI's somatosensory cortex does not process the data in the same 
manner as the control subject's. If subsequent experiments indicated that the SCI's somatosensory cortex is either functionally or anatomically atrophied, we must investigate if early rehabilitative intervention can preserve or reorganize cortical functioning.

\section{Acknowledgements}

This study was supported by the Department of Veteran Affairs Medical Research Service. We gratefully acknowledge the technical assistance of Jennifer Antick, Sherry Rascoe-Frank, and Paige Blackburn.

\section{References}

1 Zuckerman M, Hopkins TR (1966) Hallucinations or dreams? A study of arousal levels and reported visual sensations during sensory deprivation. Perceptual and Motor Skills 22: 447-459.

2 Heron W (1957) The pathology of boredom. Scientific American 196: 52-56.

3 Heron W (1961) Cognitive and physiological effects of perceptual isolation. In: P Solomon et al, editors. Sensory Deprivation. Cambridge MA: Harvard University Press, Cambridge MA: 6-33.

4 Heron W, Tait G, Smith, GK (1972) Effects of prolonged isolation of the human electroencephalogram. Brain Res 43: 280-284.

5 Zubek JP, Hughes GR, Shephard JM (1971). A comparison of the effects of prolonged sensory deprivation and perceptual deprivation. Can J Behav Sci 3: 282-290.

6 Zubek JP, Welch G (1963) Electroencephalographic changes after prolonged sensory and perceptual deprivation. Science 139: 1209-1210.

7 Braunstein P (1957) Perceptual changes following isolation of a limited tactual area. Unpublished thesis cited in JP Zubek (1969) Sensory Deprivation: 15 Years of Research. Appleton-Century-Crofts, New York.

8 Aftanas M, Zubek JP (1963) Effects of prolonged isolation of the skin on cutaneous sensitivity. Perceptual and Motor Skills 16: 565-571.

9 Aftanas M, Zubek JP (1963) Long-term effects following isolation of a circumscribed area of the skin. Perceptual and Motor Skills 17: 867-870.

10 Zubek JP (1969) Sensory and perceptual-motor process. In: Zubek JP, editor. Sensory Deprivation: 15 Years of Research. Appleton-Century-Crofts, New York.

11 Stavraky GW (1961) Supersensitivity Following Lesions of the Nervous System. University of Toronto Press, Toronto.

12 Zubek JP, Aftanas M, Kovach K, Wilgosh L, Winocur G (1963) Effect of severe immobilization of the body on intellectual and perceptual processes. Can J Psychol 17: 118-133.

13 Adey WR, Bors E, Porter RW (1968) EEG sleep patterns after high cervical lesions in man. Arch Neurol 19: $337-383$.

14 Itil TM, Saletu B, Marasa J (1972) Digital computer analyzed sleep electroencephalograms (sleep prints) in predicting anxiolytic properties of clorazepate dipotassium (tranxene). Curr Ther Res 14: 415-427.

15 Richards JS, Hirt M, Malamed L (1982) Spinal cord injury: a sensory restriction perspective. Arch Phys Med Rehabil 63: 195-199.

16 Hester GA (1971) Effects of functional transection of spinal cord on task performance under varied emotional conditions. Psychophysiology 8: 451-461.

17 Richards JS, Seitz MR, Eisele WA (1986) Auditory processing in spinal cord injury: A preliminary investigation from a sensory deprivation perspective. Arch Phys Med Rehabil 67: 115-117.

18 Merzenich MM, Nelson RJ, Stryker MP, Cyander MS, Schoppmann A, Zook JM (1984) Somatosensory cortical map changes following digit amputation in adult monkeys. J Compar Neurol 224: 591-605.

19 Neville H, Kutas M, Schmidt A (1982) Event-related potential studies of cerbral specialization during reading: II. Studies of congenitally deaf adults. Brain and Language 16: 316-337.

20 Neville H, Shmidt A, Kutas M (1983) Altered vision-evoked potentials in congenitally deaf adults. Brain Res 266: 127-132.

21 Regan D (1989) Human Brain Electrophysiology: Evoked Potentials and Evoked Magnetic Fields in Science and Medicine. Elsevier, New York.

22 Roth WT, Dorato KH, Kopell BS (1984) Intensity and Task effects on evoked physiological responses to noise burst. Psychophysiology 21: 488-481.

23 Goff WR, Allison T, Vaughan Jr HG (1978) The functional neuroanatomy of event-related potentials. In: Callaway E, Teuting P, Koslow S, editors. Event-related Brain Potentials in Man. Academic Press, New York: $1-79$.

24 Midha R, Fehlings MG, Tator CH, Saint-Cyr JA, Guha A (1987) Assessment of spinal cord injury by counting corticospinal and rubrospinal neurons, Brain Res 410: 299-308. 